\title{
Die Logik des Ausbruchs
}

\begin{abstract}
„We are not just dealing with germs that are too small to see; we are also dealing with structural hurdles that are too huge to see." (Penny 2020) „Epidemic diseases are not random events that afflict societies capriciously and without warning ... Every society produces its own specific vulnerabilities. To study them is to understand that society's structure, its standard of living, and its political priorities." (Snowden 2019: 7)
\end{abstract}

\section{Einleitung}

Als globale Pandemie hat Covid-19 inzwischen weltweit drastische politische Maßnahmen und grundlegende Einschnitte im gesellschaftlichen Leben provoziert. Entsprechend groß ist der Bedarf an einer unmittelbaren kritischen sozialwissenschaftlichen Einordnung. Relativ einig ist sich der unverzüglich publizierte „Schwall prägnanter Diagnosen“(Wagner 2020) in der Einordnung von Covid-19 als Katastrophe und Krise von historischem Ausmaß. Noch viel zu wenig werden hingegen derzeit die Kontinuitäten und Voreinstellungen in den Blick genommen, die den Umgang mit Covid-19 prägen. In diesem Beitrag vertreten wir die These, dass die aktuelle Krise sich vor dem Hintergrund einer bereits länger in Szenarien, Routinen, Techniken, Vorstellungen, Institutionen, Infrastrukturen und gesellschaftlichen Machtverhältnissen erprobten und eingerichteten Weise vollzieht. In diesen Prozessen wurde ein Rahmen gesetzt, innerhalb dessen ein neuartiges Virus erst als eine pandemische Katastrophe wirksam werden kann. Aktuelle Bewältigungsstrategien und Bearbeitungsweisen haben sich vor dem Hintergrund des Ausbruchs anderer Epidemien an anderen Orten und entlang bestimmter Rationalitäten und Technologien der Macht entwickelt.

Diese global zirkulierenden, strukturell voreingestellten Bearbeitungsmodi nennen wir ,Logik des Ausbruchs': Darunter verstehen wir eine gewisse Kohärenz in der aktuellen Bearbeitungsweise von Covid-19, die sich historisch herausgebildet hat. Sie wird einerseits in Programmatiken oder politischen 
Strategien selbst und andererseits aus dem materiellen Zusammenwirken von Programmatiken, Verfahren und Techniken, Institutionen et cetera gebildet. Dieses Zusammenwirken nennen wir „worlding“. Die vielfältigen Praktiken, Verfahren und Maßnahmen, mit denen die Interaktionen mit dem neuartigen Virus aufgefasst und bearbeitet werden, sind entlang einer Voreinstellung beschränkt: eine eingespurte Bewältigungsweise mit machtvollen Implikationen. Die kritische Richtung, die wir einschlagen, will nicht darauf hinaus, dass es eigentlich ganz anders ist, wir alle einer Chimäre aufsitzen und völlig falsche Maßnahmen ergreifen. Die als Covid-19-Pandemie verhandelte Situation ist eine reale Bedrohung, aber keine,Naturkatastrophe‘. Trotz aller vermeintlichen Zwangsläufigkeit ist auch die aktuelle Situation gesellschaftlich hergestellt, gedeutet und bearbeitet. In diesen strukturellen Voreinstellungen wurde letztlich die Krisenanfälligkeit erhöht. Damit schlagen wir eine Lesart vor, die sich der Fokussierung auf den Moment der Katastrophe gerade entzieht und vor allem Kontinuitäten sichtbar macht. Die als Covid-19-Pandemie verhandelte Situation ist kein für sich stehendes Ereignis, sondern macht letztlich eine Reihe infrastruktureller und systemischer Mängel, ihre globale Vernetzung sowie Formen ihrer Bewältigung sichtbar, die sich über Jahre entwickelt haben. Leitend ist dabei ein Blick zweiter Ordnung, der nicht nach der Verbreitung und Bekämpfung des Virus fragt, sondern nach den Strukturen, die der Verbreitung und Bekämpfung des Virus ihre ganz spezifische Form und Topologie geben.

Ist nach dem „Schwall prägnanter Diagnosen“ über den Sommer 2020 dafür immer noch Bedarf? Nach unserem Dafürhalten ist eine Perspektive auf solche Verhältnisse und Entwicklungen, die einerseits die Krisenanfälligkeit hervorbringen und andererseits die Bezugsfolie für die Bewältigung der Situation bieten, bisher noch zu wenig expliziert worden (Reckwitz 2020). Deshalb greifen wir für unser Argument im Folgenden auf die Perspektive der Kritischen Sozialepidemiologie zurück. Die Kritische Sozialepidemiologie legt den Schwerpunkt auf die sozialen und gesellschaftlichen Ursprünge von Krankheiten und vertritt die These, dass viele vermeintlich biologische Kategorien bei genauerem Hinsehen soziale sind.

Unser Beitrag reiht sich in eine wachsende Reihe von Diagnosen, welche die Ausnahmesituation vor allem als ein Schlaglicht auf bestehende strukturelle Bedingungen und Missstände thematisieren. Keineswegs ist die Krankheit der ,große Gleichmacher'. Stattdessen spielen bestehende strukturelle Ungleichheiten eine entscheidende Rolle für die Betroffenheit, sei es in Bezug auf Gender (Frey 2020), Alter (van Dyk et al. 2020) oder Diskriminierung aufgrund von Hautfarbe (Kirby 2020). Zudem werden mit der Ausnahmesituation wie durch ein Brennglas strukturelle Benachteiligungen in vielen gesellschaftlichen Bereichen sichtbar, unter anderem im Gesundheitssystem (Neely/Lopez 2020), in Städten (McFarlane 2020; Connolly et al. 2020), aufWohnungsmärkten (Holder 2020) und in der Organisation der globalen Arbeitsteilung (Harvey 2020; Kreilinger/Zeller 2020). Schließlich fordert die Situation zur Sondierung der grundlegenden gesellschaftlichen Verfasstheit heraus und legt ein neues Nachdenken nahe über die politisch-ökologischen Grundlagen von Gesundheit (Krüger/Geiselhart 2020). Inwiefern wird eine sonst hintergründige Bio- und Bevölkerungspolitik nun offen sichtbar (Lancione/Simone 2020; Hannah et al. 2020)? 
Inwiefern spinnen sich hier Regierungsweisen des Neoliberalismus wie zum Beispiel Austerität fort (Beisel et al. 2020)?

Noch wenig beleuchtet ist hingegen die Rolle von Strukturen anderer Art. Die Auseinandersetzung mit einer zu erwartenden pandemischen Infektion war bereits während der vergangenen Jahrzehnte ein Aspekt bevölkerungsmedizinischer und gesundheitspolitischer Debatten. Andreas Reckwitz hat die Bewältigung von Covid-19 jüngst als weiteren Fall einer regelmäßigen „Risikopolitik“ der Spätmoderne gedeutet (Reckwitz 2020). Arbeiten aus der Medizinanthropologie, der critical global health studies, der Sicherheitsforschung sowie der Medizingeschichte haben vielfach machtvolle Voreinstellungen, Strukturen und Programme herausgearbeitet, welche den Umgang mit Gesundheit prägen, etwa in Form der Neoliberalisierung von global health, der Versicherheitlichung von Gesundheit (Weir/ Mykhalovskiy 2010) oder eines dominanten Gefahrendiskurses der ,Emer-

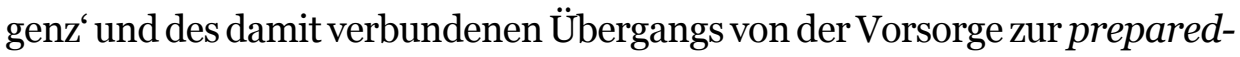
ness (Lakoff 2017; MacPhail 2010; Lynteris 2020).

An diese Arbeiten möchten wir mit einer geographischen Perspektive anschließen und anhand der aktuellen Situation solche strukturellen Kontinuitäten markieren, die den Umgang mit SARS-CoV-II mitprägen.[1] Mit einer Sensibilität für geographische Unterschiede und Brüche wird einerseits deutlich, inwiefern sich bestimmte Voreinstellungen trotz der divergierenden gesellschaftlichen Kontexte übergreifend geltend machen. Andererseits wird auch die Kontingenz der aktuellen Bewältigungsweise deutlicher erkennbar. So zeigt der Umgang mit anderen Epi- und Pandemien wie zum Beispiel AIDS, SARS oder Ebola an verschiedenen Orten der Welt auch die Bedeutung alternativer Verständnisse von Gesundheit, Gesundheitsrisiken und deren Bearbeitung. Auf dieser Basis gewinnen wir einen Standpunkt, um die Art und Weise der Problemstellung, die mit der Bewältigung verbunden ist, kritisch zu hinterfragen.

Nach einer kurzen Einführung in unsere konzeptionellen Perspektiven (2.) werden wir mit der Figur „Logik des Ausbruchs“ (3.) eine kritische Perspektive auf die aktuelle Bewältigung der Pandemie formulieren. Abschließend stellen wir konkrete Forderungen heraus, die sich aus dieser Analyse ergeben und die zur Frage führen, wie eine inklusivere Aushandlung der Auffassung von gesundheitlichen Bedingungen und deren Bearbeitung gewährleistet werden kann (4.).

\section{Kritische Sozialepidemiologie und worlding health}

Einen Ausgangs- und Anknüpfungspunkt für unser Argument liefert die Kritische Sozialepidemiologie, ein Ansatz, der in letzter Zeit vor allem in Lateinamerika entwickelt und diskutiert wird. Epidemiologie zielt allgemein auf die Erklärung des Gesundheitsgeschehens in der Bevölkerung, die Sozialepidemiologie aus dem Globalen Norden bestimmt die sozialen Determinanten von Gesundheit in erster Linie - quantitativ - als Risikofaktoren (Berkman et al. 2014). Die Kritische Sozialepidemiologie hingegen betont die sozialen und gesellschaftlichen Ursprünge von Krankheiten und stellt den verursachenden Rahmen für ungleiche Gesundheitschancen in den Vordergrund (Spiegel et al. 2015: 10; Dzudzek/Strüver 2020). Sie 
analysiert die sozialen Strukturen, die die sozialen Determinanten von Gesundheit hervorbringen (Spiegel et al. 2015: 10), also die Strukturen, die zu Armut, Arbeitslosigkeit, Rassismus und Ausschluss führen. Viele vermeintlich biologische Kategorien im Kontext von Medizin, Krankheit und Gesundheit - so die Hypothese - sind bei genauerem Hinsehen sozialer Natur (Spiegel et al. 2015; Breilh 2008; Krieger 2001). Jamie Breilh spricht - in Anlehnung an David Harvey - von einer Politischen Ökonomie der Gesundheit, die von Machtstrukturen durchzogen ist. Damit verfolgt die kritische Sozialepidemiologie einen intersektionalen Ansatz, der die Ungleichverteilung von Macht zwischen Klassen, Ethnien und Geschlecht reflektiert (Breilh 2008: 748). Und sie begreift Gesundheit selbst konsequent als eine biosoziale Tatsache. Soziale Strukturen sind nicht nur der Rahmen für Gesundheit und Krankheit, sondern Momente der Herstellung solcher Tatsachen selbst. „[W]e can begin to elucidate population patterns of health, disease and well-being as biological expressions of social relations, and can likewise begin to see how social relations influence our most basic understandings of biology." (Krieger 2001: 672)

Die konsequente Annahme einer sozialen Herstellung des Gesundheitsgeschehens hilft auch, die aktuelle Situation nicht selbstverständlich oder notwendigerweise als etwas ,zwingend' oder ,natürlich ' Gegebenes zu verstehen, sondern als etwas von der Art der Auffassung entscheidend Mitgeprägtes. Mit der Perspektive der Kritischen Sozialepidemiologie sind das Virus und die Covid-19-Infektion systematisch als biosoziale Tatsachen zu sehen, die nicht getrennt von den Prozessen und Strukturen der Hervorbringung gedacht werden können. Eine wichtige Rolle bei dieser Hervorbringung kommt der Art und Weise der Objektivierung als Krise und der daraus resultierenden Bearbeitungsweise zu. ,[B]eing vigilant on how a space of emergency is arranged - and on its implications - is as important as washing our hands to stay alive in the times that we currently inhabit." (Lancione/Simone 2020) Um das spezifische Arrangement des zu bearbeitenden Problems, um Konturen der Bearbeitungsweise und um die Performanz dieser Bearbeitungsweise bei der Hervorbringung der Krise in ihrer aktuellen Form geht es uns im Folgenden. Das Argument lautet, dass mit der Bewältigungsweise das zu Bewältigende in Teilen mitbestimmt wird und dass dieser Zusammenhang ein wichtiges Moment des Politischen ausmacht.

Unser Argument greift damit auf die grundsätzliche Annahme relationaler Ontologien zurück, das heißt die Bereitschaft, statt von einer festen Realität von der relationalen Herstellung verschiedener sozialer Welten auszugehen. In einer solchen technikphilosophisch inspirierten Perspektive werden Beziehungen als Ausgangspunkt allen Seins an den Anfang gestellt: „Beings do not preexist their relations.“ (Haraway 2003: 6) Letztlich sind soziale und auch natürliche Gegebenheiten gleichermaßen als ein Effekt von Beziehungen zu verstehen. Eine Gegebenheit ist immer auch die kontingente Stabilisierung einer bestimmten Art und Weise einer kollektiven Erfahrung. Mit der Setzung einer vermeintlich festen Natur wird diese Konstitution aus Relationen aber ausgeblendet (Descola 2010; Tsing 2015).

In der anthropologischen Debatte wird diese theoretische Grundannahme relationaler Ontologien mit Hilfe des Begriffs „worlding“ für empirische Analysen fruchtbar gemacht. Mit diesem Begriff soll das letztlich Realität 
setzende Moment betont werden, das von den aufeinander bezogenen Praktiken einer sozialen Gemeinschaft ausgeht.

Worlding macht analytisch zugänglich, dass Objekte und Ereignisse nicht für sich genommen existieren, sondern in vielfältigen Bezugnahmen ,in Dienst genommen' werden und dabei eine je eigene Welt entfalten. Hier erklärt sich die paradoxe Behauptung von Ontologien im Plural, auf der diese Perspektive beruht. Es gibt nicht ein feststehendes Ding, das sich verschieden begreifen lässt, sondern je nach worlding wird das Ding selbst anders hervorgebracht.

Im Folgenden nehmen wir eine von diesen Überlegungen inspirierte Perspektive auf die aktuelle Krise ein. Der gesellschaftliche Umgang mit dem neuartigen Phänomen SARS-CoV-II lässt sich beschreiben als eine durch aufeinander bezogene Praktiken hervorgebrachte Konstellation von anfälligen und immunisierten Körpern, gefährlichen und ungefährlichen Interaktionen, Infrastrukturen, Viren und Immunreaktionen. Vor allem werden mit dieser Perspektive bestimmte Merkmale dieses worlding thematisierbar. In Anlehnung an Annemarie Mol sprechen wir hier von einer Logik, in unserem Fall konkret von einer ,Logik des Ausbruchs', die den auf das SARS-CoV-II bezogenen Praktiken eine gewisse Kohärenz gibt, aber als durchaus fragil und nicht durchgehend konsistent vorgestellt werden soll. In diesem gerichteten, aber unabgeschlossenen Sinn verwendet auch Mol den Begriff „Logik“: „Here the term ,logic ' helps. [...] It invites the exploration of what is appropriate or logical to do in some site or situation, and what is not. It seeks a local, fragile and yet pertinent coherence." (Mol 2008: 8) „Logik des Ausbruchs“ meint bei uns das Resultat unserer Befragung der Bearbeitungsweise der Covid-19-Infektion. Eine Bearbeitungsweise, die wir nicht nur mit bestimmten Programmatiken oder politischen Strategien selbst identifizieren, sondern die aus dem materiellen worlding und dem Zusammenwirken von solchen Programmatiken, Verfahren und Techniken, Institutionen, et cetera hervorgeht: „This coherence is not necessarily obvious to the people involved. It needs not even be verbally available to them. It may be implicit: embedded in practices, buildings, habits and machines.“ (Mol 2008: 8)

Mit dem Terminus „Logik des Ausbruchs“ geht es uns um eine solche Kohärenz, auf die sich Praktiken, Verfahren und Maßnahmen beziehen und in diesen Interaktionen das neuartige Virus mit hervorbringen (worlding). Dabei bekommen die Bestandteile der Interaktion ganz reale Relevanz. Das Virus ist Welt geworden mit teils existentiellen Konsequenzen. ,Logik‘ soll dabei nicht suggerieren, die aktuelle Bearbeitungsweise sei logisch zwingend oder das einzig rational Mögliche. Der Begriff soll keine Systematik oder Zwangsläufigkeit implizieren, sondern lediglich eine gewisse Kohärenz unterschiedlicher Momente anzeigen. Es bleiben immer auch Spielräume für andere Arten des worlding. In der Wiederholung von Praktiken tritt in der Regel immer auch Unerwartetes und Kreatives hervor. Und trotzdem sind manche Sets von Praktiken kohärenter als andere. Das ist das, was der Begriff Logik evozieren soll (vgl. Mol 2008: 8). 


\section{Logik des Ausbruchs}

Die „Logik des Ausbruchs“ hat viele Facetten. Wir beschränken uns im Folgenden auf zwei dominante Momente dieser Logik. Aus unserer Perspektive zweiter Ordnung wird sowohl in der Auffassung als auch in der Bearbeitung der Interaktion mit dem neuartigen Virus ein eingespurtes Raster wirksam. Den Problembeschreibungen, ergriffenen Maßnahmen und zum Einsatz gebrachten Werkzeugen liegt eine Auffassung des Virus als Feind (3.1) zu Grunde. Daran angeknüpft wird eine Bearbeitung vor allem in der Form nationaler Sicherheitspolitik (3.2) unternommen. Die Interaktion wird damit in einer spezifischen und folgenreichen Weise hergestellt und Alternativen undenkbar gemacht.

\subsection{Klassifikation - Feind Erreger}

„Wir befinden uns im Krieg“ - das wird der französische Staatspräsident Emmanuel Macron im März 2020 nicht müde zu betonen. Der chinesische Vizepremier Sun Chunlan sagte bei seinem Besuch in Wuhan, im Kampf gegen Covid-19 dürfe es „keine Deserteure geben, sonst werden sie für immer an die Säule der historischen Schande genagelt“ (zit. n. Heywood 2020). Der ,Kampf gegen das Virus“ ist das Credo vieler anderer amtlicher Verlautbarungen, Presseberichte und Kommentare. Die gegenwärtige Notlage macht eine solche Rhetorik des Kampfes extrem plausibel. Oberste Priorität hat die Reduzierung der Infektionszahlen. Alle Anstrengungen richten sich auf die Begrenzung der Ausbreitung, die Zerstörung aller Vorkommnisse des Virus durch Desinfektion, die Entwicklung medizinischer Verfahren zur Störung seiner Replikation.

Verstärkt wird eine solche ausschließliche Fokussierung auf das Virus als Gegner durch eine etablierte Form des Umgangs mit unerwünschten Zukünften, die Adi Ophir als einmal als „catastrophization“ markiert hat (Ophir 2010). Durch das Herangehen an eine Situation, im Sinne einer Katastrophe“ wird vor allem politische Handlungsfähigkeit angesichts einer ungewissen Situation angestrebt. Erreicht wird diese Handlungsfähigkeit durch die Engführung einer komplexen Situation auf ein abgrenzbares, identifizierund bekämpfbares Objekt. ,[C]atastrophization often structures the discourse of governmentality and imposes its focal point of attention." (Ophir 2010:44) Im Falle der Covid-19-Situation ist dieser Fokus der Aufmerksamkeit das Virus. Folgt man dem Gedanken, ist diese Fokussierung womöglich zum erheblichen Teil einer Suche nach politischer Steuerungsfähigkeit geschuldet. Das Virus wird zu einem isolierbaren, feindlichen Gegenüber und damit vermeintlich kontrollierbar, allerdings unter Ausblendung eines biologisch weitaus komplexeren Verhältnisses.

Die Infektion als ein zu bekämpfender Eindringling, als ein äußerlicher Feind, ist dabei ein typisches Raster modernen Denkens. Die Idee der Hygiene selbst ist bereits verknüpft mit dem Gedanken der Ausrottung von übertragbaren Krankheiten. Die Medizinanthropologie arbeitet ferner heraus, dass unsere Vorstellungen von Biologie und Medizin in Form von Viren und Bakterien eng mit der Entwicklung der Technologien verwoben sind, die sie hervorbringt: 
„[B]iomedical technologies, particularly those that have emerged over the past century as a result of advances in molecular biology, transform, first of all, ,what it is to be biological' and hence what it is to be human. These formulations are very helpful, and we draw on them to argue that technologies should be understood as both produced through culture and as productive of culture." (Lock/Nguyen 2010: 23)

Die Katastrophisierung führt also dazu, dass das Virus als Feind mobilisiert wird. Dadurch geraten andere Erkenntnisse aus der biologischen Forschung aus dem Blick. Forschung zur Mensch-Virus-Interaktion verweist darauf, dass die unbedingte Einordnung von Viren in ein binäres Freund-FeindSchema erhebliche Verkürzungen mit sich bringt. Die Forschung weist auf die große Zahl an Viren hin, mit denen wir beständig konfrontiert sind, die Mehrheit davon ohne erkennbaren schädlichen Einfluss. „But, in spite of the parasitic nature of viruses, the human virome rarely presents any critical threat to the human organism as the great majority of such viruses target bacteria as their effective hosts." (García-López et al. 2019: 374) Als ein Kommunikations- und Distributionsmittel für genetische Informationen sind Viren nicht automatisch eine Gefahr für den Organismus; oftmals ermöglichen sie die Entstehung und Entwicklung von Leben. „[V]iruses are no more ,germs' and ,enemies' than are bacteria or human cells [...] Viruses are sources of evolutionary variation." (Margulis 1999: 82). Wie hier mit unterschiedlichen Argumenten dargestellt wird, ist demgegenüber eine Perspektive sachgerecht, welche die gegenseitige Verschränkung von Umfeld, Erreger und Mensch zu verstehen erlaubt. Die Fokussierung auf den Erreger erscheint akut plausibel, resultiert aber in einer problematischen Engführung der möglichen Bearbeitung der Situation. Die feministische Wissenschaftsforschung bietet starke Argumente, biologische und soziale Prozesse als immer schon verbundene, nicht trennbare Geschehen zu verstehen. Viren und Bakterien sind hier, wie viele andere Entitäten, wichtige nicht-menschliche Bestandteile in unseren Beziehungsgefügen. „By taking specific microbial predators as our focus we risk missing the bigger picture“, wie Mark Honigsbaum (2019: 8) zu Beginn seiner historischen Darstellung des US-amerikanischen Umgangs mit unterschiedlichen endemischen Krankheiten warnt.

Auch der zu verteidigende Körper ist in der Vorstellung des ,Virus als Eindringling ' in den individuellen Körper nur unzureichend gefasst. Wie Emily Martin in der Auseinandersetzung mit der politischen Bewältigung der Polio-Epidemie und der AIDS-Krise in den USA herausstellt, sollte körperliche Immunität nicht als ein biologisches Faktum verstanden werden. Kulturelle und politische Einflüsse schaffen erst solche mehr oder weniger stark ,immunen“ Körper (Martin 1995). „Disease is not just the outcome of a pathogenic microbe infecting a human host but emerges from socioeconomic relations, which exacerbate human-animal-microbial interactions." (Voelkner 2019: 375) Stephen Hinchliffe und andere verweisen in diesem Sinne darauf, dass sich die Entstehung einer Krankheit zumeist besser über die spezifische Immunkompetenz erklären lässt als durch das bloße Vorhandensein eines Erregers. „Being healthy may not simply mean being free from pathogens, but a matter of immunocompetence; that is, the ability 
to live with a variety of other organisms that are always in circulation." (Hinchliffe 2013: 538)

Die Isolierung des Erregers als zu bekämpfender Feind abstrahiert zudem von der Tatsache, dass die schnelle globale Zirkulation von Krankheiten konstitutiv mit der expansionistischen Logik der vorherrschenden kapitalistischen Produktionsweise verbunden ist, was zahlreiche Arbeiten aus der Mensch-Umwelt-Forschung wie der Wissenschafts- und Technikforschung bereits anhand anderer Zoonosen wie Ebola gezeigt haben (Wallace 2016; Harvey 2020; Heywood 2020). Die exzessive Expansion kapitalistischer Produktion in Ökosysteme und die Ausbreitung monokultureller Plantagenwirtschaft engt die Lebensräume bestimmter Wildtiere ein. Die Intensivierung der Landwirtschaft und insbesondere die Ausweitung der fabrikartigen Tierhaltung erleichtern zugleich Infektion und Mutation von Viren. Zudem sind die Tiere in eine weitreichende globale Warenzirkulation mit hoher Umschlagzeit eingebunden. All das schafft Bedingungen, in denen Zoonosen sehr viel schneller zirkulieren und zu einer globalen Bedrohung werden können (Chuang 2020; Wallace 2016). „Professional outbreak watchers agree that risks of a deadly pandemic are rising. [...] There are many ecological reasons for this." (Hirschfeld 2019: 3f.) Exemplarisch nennt Hirschfeld die Freisetzung alter Erreger aus schmelzendem Permafrost, Abholzung, Klimawandel, die Nischenexpansion von Arboviren (von Gliederfüßern übertragene Viren) sowie die Evolution von Antibiotikaresistenzen, die durch einen dauerhaften Einsatz von Medikamenten in der industriellen Landwirtschaft begünstigt werden.

Die lange Auseinandersetzung mit dem Mensch-Natur-Verhältnis hat vor allem auch gezeigt, dass eine dichotome Gegenüberstellung von Mensch und Natur kaum aufrecht zu halten ist (Swyngedouw 2006). Menschen greifen seit jeher in die Natur als vermeintlich unberührte Sphäre ein, zuletzt immer tiefgreifender (siehe z. B. die Anthropozän-Debatte). Die Bedingung für eine Katastrophe ist immer auch eine nicht erfolgte Bewältigung veränderter Umweltbedingungen. Dadurch sind ,Naturkatastrophen' letztlich immer gesellschaftlich mitproduziert. „Viruses mutate all of the time to be sure. But the circumstances in which mutation becomes life-threatening depend on human actions." (Harvey 2020)

Um die Interaktion mit dem neuartigen Virus zu verstehen, gilt es vor allem politische Vermittlungen in den Vordergrund zu stellen. Es ist sachgerechter, auf der Ebene der Prozesse die Bedingungen für das katastrophische Erscheinen in den Blick zu nehmen. Das als Ausdruck der Katastrophe isolierte Objekt - hier der Erreger SARS-Cov-II - ist nur eine unzureichende Annäherung an die Problemlage. Die eigentliche Krise, die es nicht erst mit der Isolierung eines neuartigen Erregers zu bewältigen gilt, spielt sich auf der Ebene gesellschaftlich gemachter ökonomischer Verhältnisse beziehungsweise von Mensch-Umwelt-Verhältnissen ab.

Infektionskrankheiten und Seuchenschutz, lange das dominierende Interesse von Tropenmedizin und später der Global-Health-Forschung, sind nur ein Aspekt des Krankheitsgeschehens. Entscheidend ist vor allem das Zusammenspiel von Infektionskrankheiten mit der gesundheitlichen Lage vor Ort, insbesondere den Umweltfaktoren und der Situation der sogenannten nicht-übertragbaren Krankheiten wie Übergewicht, Diabetes oder 
Unterernährung. Wie stark bestimmte Umwelteinflüsse, Ernährungsweisen oder Einkommensunterschiede letztlich als nicht-übertragbare Krankheiten sichtbar werden, hängt zum Beispiel stark vom Zugang zu gesundheitsfördernden Einrichtungen ab. Bezeichnend hierfür ist zum Beispiel auch die bereits jetzt überproportional festgestellte Zahl von Schwarzen unter den Todesopfern in den USA (Kendi 2020). Zentraler Punkt von Covid-19 ist der Faktor der gegenseitigen Verstärkung einzelner epidemischer Momente. Diese strukturellen gesellschaftlichen Voreinstellungen und ihre zentrale Bedeutung für das Krankheitsgeschehen geraten in der Rhetorik „Wir gegen das Virus“ und der Individualisierung von ,Risikopatient*innen“ allerdings aus dem Blick (Galea 2020).

Wir plädieren dafür, die Interaktion mit dem Virus als worlding zu begreifen, das durch spezifische Rahmenbedingungen eingespurt ist. „The characteristic microbe of a disease might be a symptom instead of a cause." (Honigsbaum 2019: 8) Mit der Thematisierung von SARS-Cov-II als tödlichem Erreger ist notwendigerweise eine Dethematisierung anderer Problematisierungen von Gesundheit und Krankheit verbunden. Die erschütternde Zahl von Toten und die teils unterschiedliche Härte, mit der die verschiedenen Gesellschaften getroffen werden, hängt sehr von den Möglichkeiten der einzelnen Gesundheitssysteme ab, Infektionen zu erkennen und Hilfsbedürftige zu versorgen. Die sozial bedingt unterschiedlichen Formen des Ausgesetztseins werden durch einzelne Vorfälle durchaus immer wieder ins öffentliche Bewusstsein gerückt. Symptomatisch ist etwa der Fall Tönnies, bei dem sich im Juni 2020 Arbeiter*innen in prekären Arbeits- und Wohnverhältnissen in einer Schweineschlachterei massenhaft infizierten. Gestützt durch die Reduzierung der Situation auf den Kampf jeder Einzelnen gegen das Virus überwiegt gleichwohl immer noch eine Individualisierung des Risikos (Adamczak 2020).

\subsection{Zurichtung - Gesundheit als nationales Sicherheitsproblem}

Die Auffassung der Interaktion mit dem neuartigen Virus in einer FreundFeind-Dichotomie wird häufig im Nationalstaat als zu schützender, Nationalkörper' verräumlicht. In enger Verbindung dazu besteht die zweite Voreinstellung einer ,Logik des Ausbruchs' in einer sicherheitspolitischen Bearbeitung des Gesundheitsgeschehens. Philipp Sarasin weist auf die Gefahren einer Vermischung bestimmter medizinischer Konzepte mit handlungsleitenden politischen Metaphern hin:

„If [...] the boundaries of a nation are metaphorically equated with the boundaries of the ,body politic' and protected against all kinds of ,infections', then the threshold between the medical-epidemiological and the metaphorical-political concepts of infection has already been transgressed in a dangerous way." (Sarasin 2008: 268)

Der Grund für die zunehmende Dethematisierung einer möglichen globallokalen Bearbeitungsweise zugunsten von Vorstellungen nationaler Sicherheit liegt in der Entwicklung von global health begründet. Mit der Gründung der WHO im Jahr 1946 wurde der „Besitz des bestmöglichen Gesundheitszustandes“ als „eines der Grundrechte jedes menschlichen Wesens, ohne 
Unterschied der Rasse [sic!], der Religion, der politischen Anschauung und der wirtschaftlichen oder sozialen Stellung“(WHO 1946: 1) festgeschrieben. Durch die „Erklärung von Alma-Ata“ (WHO 1978) erhielt das Konzept der primären Gesundheitsversorgung oberste Priorität und mit der „OttawaCharta“ wurde „Gesundheit für alle“ (HFA) zu einer Aufgabe der internationalen Gemeinschaft (WHO 1986). Die politische Errungenschaft, medizinische Grundversorgung strukturell als wirksamstes Mittel gegen Seuchen wie auch gegen andere Krankheiten zu fördern, wurde allerdings vor fast 30 Jahren aufgekündigt. Das geschah vor allem auf Betreiben der USA und anderer großer Geberländer durch das faktische Einfrieren der flexibel verfügbaren Mitgliedsbeiträge, um mit freiwilligen Zusatzzahlungen mehr direkten Einfluss auf die Aktivitäten der WHO auszuüben. Bereits vor Covid-19 beklagten Expert*innen, dass 8o Prozent des Etats der WHO aus privaten Spenden und Stiftungen gespeist wird. Dieses Geld ist in der Regel projektgebunden, und zwar in solchen Vorhaben, die im Vergleich zum strukturellen Ausbau von Gesundheitsinfrastruktur schnelle und prestigeträchtige Erfolge versprechen, wie zum Beispiel die Malaria-Bekämpfung durch die Melindaund Bill-Gates-Stiftung.

„[D]er Notfallfonds für Epidemien bei der WHO, der nach der EbolaKrise eingerichtet wurde, und der eigentlich immer mit 10o Mio. Dollar für Notfälle gefüllt sein soll, hat diese Zielmarke aufgrund der spärlichen Beiträge der Mitgliedstaaten in den letzten 5 Jahren fast nie erreicht.“ (Wulf 2020)

Diese Neoliberalisierung und chronische Unterfinanzierung der WHO hat dazu geführt, dass die Logik der Bearbeitung von Epidemien durch medizinische Grundversorgung zugunsten eines Problembearbeitungsmodus von Epidemien als Sicherheitsproblem (Lakoff/Collier 2008) abgelöst wurde. An die Stelle der Verhinderung von Seuchen rückt die Vorstellung, ,für den unvermeidlichen Ernstfall vorbereitet" sein zu müssen, um dann schnell mit Logistik und Infrastruktur reagieren zu können (preparedness). Hier kann ein Übergang von einer, universellen Logik‘, die Gesundheit als eine grundsätzliche Bedingung der Teilhabe jedes Individuums zu gewährleisten versucht, hin zu einer ,biopolitischen' Logik beobachtet werden, die vor allem nationale Bevölkerungen schützt, indem Infektionsketten unterbrochen oder der ,Nationalkörper‘ durch das Schließen von Grenzen isoliert werden soll (Lakoff 2017).

Eine auf nationale Sicherheit zielende Bearbeitungsweise von Covid-19 erweckt den Eindruck, man habe es mit einer Reihe nationaler Epidemien zu tun. Fall- und Sterbezahlen, die stets in nationalen Containern präsentiert und geographisch in Karten repräsentiert werden, verstärken diesen Eindruck. Eine stark verräumlichende und containerisierende Logik abstrahiert davon, dass das Virus global zirkuliert und kontextspezifische Antworten braucht. Es handelt sich bei Covid-19 aber um eine globale Pandemie, die vor nationalen Grenzen nicht Halt macht und vor allem gute lokale Strukturen zu ihrer Bearbeitung benötigt. Aber Vorschläge zu globalen wie lokalen Bearbeitungsweisen der Krise bleiben weitgehend ungehört.

Eine solche Setzung und Fixierung von Covid-19 als national zu bearbeitendes ,Sicherheitsproblem ' führt dazu, dass andere worldings, zum 
Beispiel lokal in anderen Teilen der Welt als spezifische Antwort auf andere Epidemien entwickelte lokale wie globale Bearbeitungsweisen, aus dem Blick geraten. Sie sind auch deshalb so wichtig, weil sie die Probleme und Dethematisierungen offenlegen, die eine Setzung und Fixierung als nationales Sicherheitsproblem mit sich bringt. Kaleidoskopartig werden wir diese anderen worldings anhand von Community-Health-Initiativen an verschiedenen Orten der Welt ablesen, die im Kampf gegen verschiedene Epidemien involviert sind.

Singapur. Bereits im Februar 2020 warnt die singapurische Non-ProfitOrganisation „transient workers count too“, Arbeitgeber*innen könnten die Covid-19-Epidemie in Singapur lostreten, sollten sie nicht damit aufhören, migrantischen Arbeiter*innen trotz Krankenschein mit Entlassung zu drohen, sofern sie nicht zur Arbeit erschienen (Lloyd C 2020). Obwohl Singapur in einer sehr frühen Phase des Ausbruchs umfassend getestet, Kontakte nachverfolgt und Quarantänen angeordnet hatte, sodass ein Lockdown zunächst nicht nötig war, schossen die Infektionszahlen Anfang März schließlich dramatisch in die Höhe. Das Virus hatte sich in der Tat unter den Wanderarbeiter*innen aus Bangladesch, Indien und China verbreitet, die räumlich segregiert leben und häufig mit wenig mehr Rechten als einer Arbeitserlaubnis ausgestattet sind (Stack 2020). Nationale, versicherheitlichte Strategien ohne den Einbezug von Menschenrechten und ein Recht auf Gesundheit für alle greifen offenbar zu kurz.

Johannesburg, Südafrika. „Mittelfristig ist Covid-19 (wie auch immer es aussehen mag) ein Argument für die Stärkung der primären Gesundheitssysteme" (Heywood 2020, siehe z. B. auch Jung/Wulf 2015; Wulf 2020; Jung 2020), kann der südafrikanische Menschenrechtsaktivist Mark Heywood bereits zu Beginn der Covid-19-Krise sagen. Denn seine Aussagen speisen sich aus jahrzehntelangen Erfahrungen im Umgang mit HIV. Die global vernetzte Bearbeitung von HIV durch Community-Health-Initiativen von unten zeigt, dass ein Virus nur mit einem Menschenrechtsansatz, der Stigmatisierungen aufgrund von Nationalität, Hautfarbe oder sexueller Orientierung et cetera vermeidet und allen Menschen gleichermaßen Zugang zu Gesundheit ermöglicht, erfolgreich eingedämmt werden kann. Wie wichtig die Vermeidung von Stigmatisierung gerade im Zuge massenhafter Tests ist, zeigt auch der Umgang mit SARS in Hong Kong (Lee et al. 2005) sowie der aktuelle Umgang mit Covid-19 in Südkorea (Chiara 2020).

Freetown, Sierra Leone; Beni und Goma, Kongo. Menschenrechtsaktivist*innen (Jung/Wulf 2015) und Sozialwissenschaftler*innen (Morisho et al. 2020; Leach 2020) zeigen, dass Bearbeitungsmodi der Krise nur dann erfolgreich sein können, wenn sie von der lokalen Bevölkerung auch umgesetzt und angewendet werden können. Dieser Umstand führt dazu, dass lokale Bearbeitungsmodi aus der Ebola-Krise, wie die Entwicklung von Strategien, trotz physischer Distanz soziale Nähe leben zu können, nun erfolgreich im Umgang mit Covid-19 eingesetzt werden können. Statt autoritärer Kontrolle ist die gemeinschaftliche Ermächtigung zu Hygiene- und Sicherheitsmaßnahmen wichtig. Abstand kann nur dann gehalten werden, wenn trotzdem Menschen mit dem Lebensnotwendigen versorgt und vor Isolation bewahrt werden können (Leach 2020). „Die Lehre aus Ebola? Gesundheitssysteme gehören in öffentliche Hand, zugänglich für alle Menschen 
und unabhängig von ihrem Einkommen. Das ist der einzig zuverlässige und nachhaltige Schutz gegen die Epidemien der Zukunft.“ (Jung 2020)

Die skizzierten globalen und zugleich lokalen Bearbeitungsweisen der Krise stellen alternative worldings dar, die im Rahmen von Covid-19 mobilisiert werden können. Sie sichtbar zu machen, erlaubt erstens, aus dem Umgang mit neuartigen Viren zu lernen. Als wissenschaftstheoretische Kategorie erlaubt die Perspektive des worldings zweitens zu zeigen, welche Voreinstellungen, welche anderen Ideen die vorherrschende Sicht auf das Covid-19-Geschehen prägen. In diesem Sinne werden wir im Fazit der Frage nachgehen, wie die Einspurung einer ,Logik des Ausbruchs' und ihre problematischen Implikationen mit alternativen Auffassungen konfrontiert werden können und damit die gegenwärtige Unbedingtheit und Zwangsläufigkeit verlieren, ohne die Interaktion mit dem Virus oder die Auswirkungen von Covid-19 zu ignorieren.

\section{Für eine solidarische Politik des Lebens}

In unserer Analyse haben wir versucht, die Grundannahme einer Kritischen Sozialepidemiologie bezüglich der sozialen Herstellung vermeintlicher biologischer Phänomene mit Hilfe von Argumenten aus Technikphilosophie und Sicherheitsforschung auf die aktuelle Covid-19-Situation zu beziehen. Mit der Kritischen Sozialepidemiologie treten wir auch für die normative Zielperspektive eines grundlegenden Rechts auf Gesundheit ein, verstanden „als Abwesenheit von systematischen Gesundheitsungleichheiten zwischen Gruppen unterschiedlicher sozialer Hierarchien“ (Spiegel et al. 2015: 11). Solche systematischen Ungleichheiten gründen nicht nur in der Verbreitung und Bekämpfung von Viren und Seuchen, sondern bereits in der Art und Weise der Auffassung und Bewältigung einer Krankheit als viraler Bedrohung, dem Ausblenden struktureller Bedingungen et cetera. Da unsere Analyse inmitten der Covid-19-Krise stattfand, ist sie weder abschließend noch vollständig, sondern vermittelt lediglich einen Überblick über prägende Strukturen. Diese sind erstens die populärwissenschaftliche Rahmung von Viren als Feinden, denen der Krieg zu erklären ist. Dieser Kampf sei dann vor allem von Einzelnen zu führen. Damit verbunden ist vor allem eine Dethematisierung von Möglichkeiten, in solidarischen Gemeinschaften ,mit dem Virus zu leben'. Zweitens zeigte sich durch die zunehmende Versicherheitlichung von Gesundheitspolitik eine Fokussierung auf nationale Problembearbeitungsmodi. Die Neoliberalisierung der WHO und nationaler Gesundheitssysteme führt dazu, dass der universelle Anspruch der Vermeidung und Heilung von Krankheiten zugunsten eines Managements von Epidemien (preparedness) verschoben wird.

Gemeinsam ist den Momenten der von uns beschriebenen ,Logik des Ausbruchs', dass die verbundenen Politiken in Form von medizinischen Handlungsanweisungen oder Versicherheitlichungen letzten Endes liberal autoritär sind. Vor diesem Hintergrund stellt sich eine Frage, die die Medizinanthropologie bereits länger stellt und die im Zuge von „Leben in kapitalistischen Ruinen“(Tsing 2015) im Anthropozän neue Brisanz erhält: Wie also kann eine demokratische Politik des Lebens aussehen, an der eine kritische und aktivistische Praxis ansetzen kann? Sind „kollektive Praktiken 
möglich [...], die tatsächlich zur Gesundheit der Bevölkerung beitragen und die auch Verhaltensänderungen im großen Maßstab umfassen, ohne gleichzeitig Formen von Zwang und Überwachung auszuweiten?" (Sotiris 2020:3) Wie kann „eine alternative Politik des bios, die individuelle und kollektive Fürsorge ohne Zwang miteinander verbindet" (ebd.), wie sie der griechische Philosoph Panagiotis Sotiris fordert, konkret aussehen? Wie können wir das worlding epidemischer Krisen kollektiv verhandeln?

Worlding bietet „eine Perspektive auf Orte, an denen globales Wissen [...] und Ungerechtigkeiten nicht nur fortgeschrieben, sondern angefochten werden können "(Biehl 2016: 135; Übers. d. A.). Damit haben wir eine geographische Perspektive vorgestellt, die es in Zukunft ermöglicht, nicht-autoritäre, solidarische, partizipative, allen Menschen verpflichtete Bearbeitungsweisen von Krisen und Praktiken sowie Gesundheitsfürsorge in globaler Perspektive zu erforschen. Eine solche Perspektive verbindet die Idee einer „Biopolitik von unten“ (Sotiris 2020) mit der Idee einer, Politik des Lebens von woanders für alle', die vor allem an Erfahrungen aus dem globalen Süden anknüpft.

Folgende Fragen für die weitere Forschung lassen sich aus unseren Überlegungen ableiten.

An die Stelle eines Krieges gegen das Virus kann die Frage treten, wie wir mit dem Virus leben können. „Wir alle sind abhängig von unterstützenden Infrastrukturen, von ökonomisch, kulturell, sozial und historisch je spezifischen Netzwerken und Bindungen und von Anerkennungsverhältnissen, die uns im Leben halten“, schreibt die feministische Soziologin Sabine Hark in Bezug auf Covid-19. „Es ist eine Abhängigkeit, die wir nicht übergehen können. Sie ist ein nicht verhandelbarer Umstand unseres Seins als körperliche Wesen. Die Pandemie macht aber auch deutlich, dass wir diese Strukturen der Unterstützung sowie die Netzwerke des Lebens dort, wo sie fehlen, auch und gerade unter der Bedingung ihres Fehlens beziehungsweise ihrer systematischen Verhinderung schaffen müssen." (Hark 2020)

An die Stelle einer Logik des Ausbruchs, die auf Sicherheit und preparedness setzt, kann die Frage treten, wie eine strukturelle Prävention gestaltet sein kann, die die medizinische Grundversorgung von communities in demokratischer Teilhabe sichert. „Die mit dem Globalisierungsprozess entstandenen Gesundheitsrisiken dürfen nicht zur Legitimation einer Politik der kurzfristigen Krisenintervention im Stil quasimilitärischer Operationen mit Luftbrücken, militärischem Sanitätspersonal und Feldlazaretten - begleitet von Wohltätigkeitsveranstaltungen und Katastrophenbildern zum Spendensammeln - dienen." (Jung/Wulf 2015: 3) Wie kann das neoliberalisierte Gesundheitssystem global wie lokal wieder in öffentliche Hände gelegt werden (Kreilinger/Zeller 2020)? Denn nur eine Gesundheitsversorgung, die niemanden aufgrund von Nationalität, Hautfarbe oder Geschlecht zurücklässt, wird das Virus erfolgreich bekämpfen.

Hierzu bedarf es erstens „,sozialer Strukturpolitiken, die lokales, insbesondere zivilgesellschaftliches, und damit kontextsensibles Wissen sowie auf die Partizipation der Betroffenen ausgerichtete Prozesse der Selbstorganisation zum Ausgangspunkt nehmen " (Jung/Wulf 2015: 3). Zweitens bedarf es einer weiteren Erforschung des worldings von Community-Health-Initiativen, die sich in unterschiedlichen Kontexten gegen Covid-19 engagieren, wie z. B. das Polikliniksyndikat, ein Verbund solidarischer Gesundheitszentren in 
Deutschland. In ihrem „Aufruf zur Stärkung solidarischer Strukturen in Zeiten der Corona-Pandemie“ (Polikliniksyndikat - Solidarische Gesundheitszentren 2020) fordert es, solidarische Nachbarschaftsstrukturen und soziale und soziokulturelle Träger finanziell zu unterstützen, damit alle Menschen ungeachtet von sozioökonomischem Status, Alter oder Herkunft so versorgt werden können, dass sie sich gemeinsam aktiv und effektiv gegen das Virus schützen können. Aufgabe eines solidarischen Gesundheitswesens ist die gemeinschaftliche Entwicklung von Strategien, die es allen Menschen ermöglichen, das Netz des Lebens sicher, würdevoll und aus physischer Distanz zu pflegen, ohne jemanden zurückzulassen (\#leavenoonebehind). Diese Maßnahmen dienen der Verlangsamung der Ausbreitungsgeschwindigkeit des Virus und der Herstellung sozialer Gerechtigkeit in gleichem Maße.

„Aus einer solchen Perspektive würden die Entscheidungen für Bewegungseinschränkungen und soziale Distanzierung während einer Epidemie [...] demokratisch diskutiert und kollektiv gefällt. Das bedeutet, dass wir von einer Haltung der bloßen Disziplin zur Verantwortung übergehen, in Bezug auf andere und dann auf uns selbst, und von einer Aussetzung der Sozialität zu ihrer bewussten Transformation. In einem solchen Zustand bewegen wir uns von einer permanenten individualisierten Angst, in der jeglicher sozialer Zusammenhalt zerbrechen kann, zu einer Idee kollektiver Anstrengung, Koordination und Solidarität in einem gemeinsamen Kampf.“ (Sotiris 2020: 3)

Anstatt die erfolgreiche Bekämpfung von Covid-19 in anderen Teilen der Welt als „zivilisatorische Kränkung“ aufzufassen (Siemons 2020), bietet es sich hier an, aus anderen Teilen der Welt, die bereits konkrete Erfahrungen im Umgang mit Epidemien gemacht haben, zu lernen. Eine Geographie und Kritische Sozialepidemiologie, die die Vielfältigkeit der worldings von global health aufzeigen, können hier einen Anfang bilden.

Dieser Artikel wurde durch den Open-Access-Publikationsfonds der Universität Münster gefördert.

\section{Endnoten}

[1] Wir danken den Teilnehmer*innen der Gedankenräume in Münster und dem KuSo-Forum in Berlin sehr herzlich für das wertvolle Feedback zu diesem Artikel. Ein Teil der diesem Artikel zugrundeliegenden Überlegungen stammen aus dem Projekt „Worlding Medicine“, das zwischen 2018 und 2021 von der Deutschen Forschungsgemeinschaft gefördert wird (Projektnummer 392750976). Die Projektleiterin dankt der DFG sehr herzlich für die Unterstützung des Projekts sowie projektbegleitender Workshops, die die Kooperation für den vorliegenden Artikel ermöglicht haben.

\section{Autor innen}

Henning Füller setzt sich in seiner Forschung mit dem Verhältnis von Macht, Raum und Technik auseinander, zuletzt am Gegenstand Public Health und Infektionskontrolle.

henning.fueller@geo.hu-berlin.de 
Iris Dzudzek ist Humangeographin und arbeitet zu Fragen von Macht und Wissen im Spannungsfeld von Globalisierung, Stadt und Gesundheit.

iris.dzudzek@uni-muenster.de

\section{Literatur}

Adamczak, Bini (2020): Von Menschen, Fledermäusen und Göttern. Das Virus verbindet und trennt. Kritik an der Individualisierung von Risiko. https://www.neues-deutschland. de/artikel/1139276.corona-und-soziale-folgen-von-menschen-fledermaeusen-undgoettern.html (letzter Zugriff am 3.8.2020).

Beisel, Uli / Glasman, Joël / Ouma, Stefan (2020): Genesene Menschen als Ressource? Gastbeitrag. https://www.fr.de/wissen/genesene-menschen-ressource-13747157.html (letzter Zugriff am 1.5.2020).

Berkman, Lisa F. / Kawachi, Ichiro / Glymour, Maria M. (2014): Social Epidemiology. Oxford: Oxford University Press.

Biehl, Joao (2016): Theorizing global health. In: Medicine Anthropology Theory. An openaccess journal in the anthropology of health, illness, and medicine 3/2, 127-142.

Breilh, Jaime (2008): Latin American critical (,Social') epidemiology: new settings for an old dream. In: International Journal of Epidemiology 37/4, 745-750.

Chuăng (2020): Soziale Ansteckung. Mikrobiologischer Klassenkampf in China. In: a \& k 658. https://www.akweb.de/politik/soziale-ansteckung/ (letzter Zugriff am 25.10.2020).

Connolly, Creighton / Ali, Harris / Keil, Roger (2020): The Urbanization of COVID-19. https://urbanpolitical.podigee.io/16-covid19 (letzter Zugriff am 14.3.2020).

Descola, Philippe (2010): Cognition, Perception and Worlding. In: Interdisciplinary Science Reviews 35/3-4, 334-340.

van Dyk, Silke / Graefe, Stefanie / Haubner, Tine (2020): Das Überleben der „Anderen“: Alter in der Pandemie. In: Blätter für deutsche und internationale Politik 5, 33-36.

Dzudzek, Iris / Strüver, Anke (2020): Urbane Gesundheitsgerechtigkeit. Öko-sozialepidemiologische Forschungsperspektiven für eine Kritische Stadtgeographie verkörperter Ungleichheiten. In: Geographische Zeitschrift, online first. DOI 10.25162/gz-2020-0005.

Frey, Regina (2020): Corona und Gender - ein geschlechtsbezogener Blick auf die Pandemie und ihre (möglichen) Folgen. https://www.gender.de/cms-gender/wp-content/uploads/ gender_corona.pdf (letzter Zugriff am 20.3.2020).

Galea, Sandro (2020): The Poor and Marginalized Will Be the Hardest Hit by Coronavirus. https://blogs.scientificamerican.com/observations/the-poor-and-marginalized-will-bethe-hardest-hit-by-coronavirus (letzter Zugriff am 15.9.2020).

García-López, Rodrigo / Pérez-Brocal, Vicente / Moya, Andrés (2019): Beyond cells - The virome in the human holobiont. In: Microbial Cell 6/9, 373-396.

Hannah, Matthew G. / Hutta, Jan Simon / Schemann, Christoph (2020): Thinking Through Covid-19 Responses with Foucault - An Initial Overview. https://antipodeonline. org/2020/05/05/thinking-through-covid-19-responses-with-foucault/ (letzter Zugriff am 3.5.2020).

Haraway, Donna (2003): The Companion Species Manifesto: Dogs, People and Significant Otherness. Boulder/London: Paradigm.

Hark, Sabine (2020): Die Netzwerke des Lebens. In: Frankfurter Rundschau, 3.4.2020.

Harvey, David (2020): Anti-Capitalist Politics in the Time of COVID-19. http://davidharvey.org/2020/03/anti-capitalist-politics-in-the-time-of-covid-19/ (letzter Zugriff am 22.3.2020).

Heywood, Mark (2020): Die autoritäre Versuchung. medico international. https://www. medico.de/die-autoritaere-versuchung-17665/ (letzter Zugriff am 24.4.2020).

Hinchliffe, Steve / Allen, John / Lavau, Stephanie / Bingham, Nick / Carter, Simon (2013): Biosecurity and the topologies of infected life. From borderlines to borderlands. In: Transactions of the Institute of British Geographers 38/4, 531-543.

Hirschfeld, Katherine (2019): Microbial insurgency: Theorizing global health in the Anthropocene. In: The Anthropocene Review 7/1, 3-18.

Holder, Sarah (2020): What Cities Are Doing to Stall Evictions and Foreclosures. https:// www.citylab.com/equity/2020/o3/covid-19-housing-security-eviction-utility-shut-offmortgage/607951/ (letzter Zugriff am 23.3.2020). 
Honigsbaum, Mark (2019): The Pandemic Century. London/New York: W. W. Norton.

Jung, Anne (2020): Von Ebola lernen - global handeln. https://www.medico.de/blog/ global-handeln-17690/ (letzter Zugriff am 22.5.2020).

Jung, Anne / Wulf, Andreas (2015): Terrorismus der Armut. In: iz3w 347, 18-20.

Kendi, Ibram X. (2020): What the Racial Data Show. https://www.theatlantic.com/ideas/ archive/2020/04/coronavirus-exposing-our-racial-divides/609526/ (letzter Zugriff am 7.4.2020).

Kirby, Tony (2020): Evidence mounts on the disproportionate effect of COVID-19 on ethnic minorities. In: The Lancet Respiratory Medicine 8/6, 547-548.

Kreilinger, Verena / Zeller, Christian (2020): Corona-Pandemie - eine historische Wende. Aufbruch für ökosozialistische Alternative. http://www.oekosoz.org/2020/o3/coronapandemie-eine-historische-wende/ (letzter Zugriff am 22.5.2020).

Krieger, Nancy (2001): Theories for social epidemiology in the 21st century: an ecosocial perspective. In: International Journal of Epidemiology 30/4, 668-677.

Krüger, Fred / Geiselhart, Klaus (2020): Das Corona-Virus und die Politische Ökologie von Gesundheit. https://www.geographie.nat.fau.de/das-corona-virus-und-die-politischeoekologie-von-gesundheit/ (letzter Zugriff am 15·5.2020).

Lakoff, Andrew (2017): Unprepared. Global Health in a time of emergency. Oakland: University of California Press.

Lakoff, Andrew / Collier, Stephen J. (2008): Biosecurity Interventions. New York: Columbia University Press.

Lancione, Michele / Simone, AbdouMaliq (2020): Bio-austerity and Solidarity in the Covid-19 Space of Emergency - Episode One. https://www.societyandspace.org/articles/ bio-austerity-and-solidarity-in-the-covid-19-space-of-emergency (letzter Zugriff am 17.4.2020).

Leach, Melissa (2020): Echoes of Ebola: social and political warnings for the COVID-19 response in African settings. http://somatosphere.net/forumpost/echoes-of-ebola/ (letzter Zugriff am 25.10.2020).

Lee, Sing / Chan, Lydia Y. Y. / Chau, Annie M. Y. / Kwok, Kathleen P. S. / Kleinman, Arthur (2005): The experience of SARS-related stigma at Amoy Gardens. In: Social Science \& Medicine 61/9, 2038-2046.

Lloyd C (2020): How employers can kickstart an epidemic. http://twc2.org.sg/2020/o3/o1/ how-employers-can-kickstart-an-epidemic/ (letzter Zugriff am 1.5.2020).

Lock, Margaret M. / Nguyen, Vinh-Kim (2010): An anthropology of biomedicine. London: Wiley-Blackwell.

Lynteris, Christos (2020): Human Extinction and the Pandemic Imaginary. London: Routledge.

MacPhail, Theresa (2010): A Predictable Unpredictability. The 2009 H1N1 pandemic and the concept of strategic uncertainty within global public health. In: Behemoth 1/3, 5-77.

Margulis, Lynn (1999): Symbiotic Planet: A New Look at Evolution. London: Phoenix.

Martin, Emily (1995): Flexible Bodies: Tracking Immunity in American Culture from the Days of Polio to the Age of AIDS. Boston: Beacon.

McFarlane, Colin (2020): The urban poor have been hit hard by coronavirus. We must ask who cities are designed to serve. https://theconversation.com/the-urban-poor-havebeen-hit-hard-by-coronavirus-we-must-ask-who-cities-are-designed-to-serve-138707 (letzter Zugriff am 8.6.2020).

Mol, Annemarie (2008): The Logic of Care: Health and the Problem of Patient Choice. London: Routledge.

Morisho, Nene / Kalubi, Josepha / Park, Sung-Joon / Doevenspeck, Martin (2020): Same but Different? A Comparison of Ebola Virus Disease and Covid-19 After the Ebola Epidemic in Eastern DRC (2018-20). https://africanarguments.org/2020/04/24/same-but-differenta-comparison-of-ebola-virus-disease-and-covid-19-after-the-ebola-epidemic-in-easterndrc-2018-20/ (letzter Zugriff am 22.5.2020).

Neely, A. / Lopez, Patricia (2020): Care in Time of Covid-19. https://antipodeonline. org/2020/03/10/care-in-the-time-of-covid-19/ (letzter Zugriff am 1.6.2020).

Ophir, Adi (2010): The Politics of Catastrophization: Emergency and Exception. In: Didier Fassin / Mariella Pandolfi (Hg.), Contemporary states of emergency. New York: Zone Books, 40-61.

Penny, Laurie (2020): Panic, Pandemic, and the Body Politic. https://www.wired.com/ story/what-coronavirus-pandemic-says-about-society/ (letzter Zugriff am 24.4.2020). 
Polikliniksyndikat - Solidarische Gesundheitszentren (2020): Aufruf zur Stärkung solidarischer Strukturen in Zeiten der Corona-Pandemie. http://poliklinik1.org/news/202004-03/aufruf-zur-staerkung-solidarischer-strukturen-zeiten-der-corona-pandemie (letzter Zugriff am 22.5.2020).

Reckwitz, Andreas (2020): Verblendet vom Augenblick. In: Die Zeit, 10.6.2020.

Sarasin, Philipp (2008): Vapors, Viruses, Resistance(se): The Trace of Infection in the Work of Michel Foucault. In: Roger Keil / Syed Ali Harris (Hg.), Networked Disease. Hoboken: Wiley-Blackwell, 267-280.

Siemons, Mark (2020): Corona und der Westen: Die zivilisatorische Kränkung. https:// www.faz.net/aktuell/feuilleton/debatten/corona-und-der-westen-die-zivilisatorischekraenkung-16700907.html (letzter Zugriff am 22.5.2020).

Snowden, Frank M. (2019): Epidemics and Society. New Haven: Yale University Press.

Sotiris, Panagiotis (2020): Ist eine demokratische Biopolitik möglich? In: LuXemburg Online.

Spiegel, Jerry M. / Breilh, Jaime / Yassi, Annalee (2015): Why language matters: insights and challenges in applying a social determination of health approach in a North-South collaborative research program. In: Globalization and Health 11/1, 1-17.

Stack, Megan K. (2020): A Sudden Coronavirus Surge Brought Out Singapore's Dark Side. https://www.nytimes.com/2020/05/20/magazine/singapore-coronavirus.html (letzter Zugriff am 21.5.2020).

Swyngedouw, Erik (2006): Metabolic urbanization: The making of cyborg cities. In: Nikolas Heynen / Maria Kaika (Hg.), In the Nature of Cities. Urban Political Ecology and the Politics of Urban Metabolism. London: Routledge, 21-40.

Tsing, Anna Lowenhaupt (2015): The Mushroom at the End of the World. Princeton: Princeton University Press.

Voelkner, Nadine (2019): Riding the Shi: From Infection Barriers to the Microbial City. In: International Political Sociology 13/4, 375-391.

Wagner, Peter (2020): Wissen, um rechtzeitig angemessen zu handeln. https://soziopolis. de/beobachten/gesellschaft/artikel/wissen-um-rechtzeitig-angemessen-zu-handeln/ (letzter Zugriff am 7.5.2020).

Wallace, Robert G. (2016): Big Farms Make Big Flu. Dispatches on Infectious Disease, Agribusiness and the Nature of Science. New York: Monthly Review Press.

WHO (1946): Constitution of the World Health Organisation. New York: World Health Organization.

WHO (1978): Declaration of Alma-Ata: International Conference on Primary Health Care, Alma-Ata, USSR, 6.-12. September 1978. Alma-Ata: World Health Organization.

WHO (1986): Ottawa Charter for Health Promotion. Ottawa: World Health Organization.

Wulf, Andreas (2020): Corona-Virus - Quarantäne mit Augenmaß? https://www.medico. $\mathrm{de} / \mathrm{blog}$ /quarantaene-mit-augenmass-17650/ (letzter Zugriff am 22.5.2020). 
